\title{
Representing energy. II. Energy tracking representations
}

\author{
Rachel E. Scherr, ${ }^{1}$ Hunter G. Close, ${ }^{2}$ Eleanor W. Close, ${ }^{2}$ and Stamatis Vokos ${ }^{1}$ \\ ${ }^{1}$ Department of Physics, Seattle Pacific University, Seattle, Washington 98119, USA \\ ${ }^{2}$ Department of Physics, Texas State University, San Marcos, Texas 78666, USA
}

(Received 11 July 2012; published 19 October 2012)

\begin{abstract}
The Energy Project at Seattle Pacific University has developed representations that embody the substance metaphor and support learners in conserving and tracking energy as it flows from object to object and changes form. Such representations enable detailed modeling of energy dynamics in complex physical processes. We assess student learning by means of representations that learners invent to explain energy dynamics in specific real-world scenarios. Refined versions of these learner-generated representations have proven valuable for our own teaching, physics understanding, and research.
\end{abstract}

\section{INTRODUCTION}

We have identified a substance ontology for energy as having special advantages for physics instruction in which the emphasis is on conceptual understanding of local energy conservation, transfer, and flow. Classic representations of energy, such as bar charts and pie charts, all represent energy to some degree as being conserved, localized, and changing form, but have limited utility for tracking the flow of energy among objects [1].

After describing our instructional context and learning goals (Sec. II), we introduce a specific class of representations that embody the substance metaphor and strongly promote energy conservation and tracking (Sec. III). We explore the unique affordances of one of these representations in which the coordinated use of several human bodies is a context for transparent communal thinking. We assess student learning by means of representations that learners invent to show energy dynamics in specific real-world scenarios (Sec. IV). Refined versions of these learnergenerated representations (described in Sec. V) have proven valuable for our own teaching, physics understanding, and research.

\section{CONTEXT FOR INVESTIGATION}

\section{A. The Energy Project}

The Energy Project is a five-year NSF-funded project whose goal is to promote elementary and secondary teachers' development of formative assessment practices in the context of energy. When challenged by the needs of our local community to provide professional development courses for in-service teachers on energy, we recognized the pedagogical incompatibility between our own familiar

Published by the American Physical Society under the terms of the Creative Commons Attribution 3.0 License. Further distribution of this work must maintain attribution to the author(s) and the published article's title, journal citation, and DOI. instructional paradigm and the understanding of energy that we hoped for our community of teachers to achieve, both in extent and character. On one hand, our existing instructional expertise prepared us to lead teachers to engage in guided, linear sequences of logic within the borders of carefully prechosen, simplified physical scenarios-those in which we, the instructors, could easily limit the topics of teachercontributed discourse to conceptual issues for which we had prepared. On the other hand, our goals for teachers included that they would recognize the universal applicability of the theory of energy beyond any short list of scenarios, and beyond "school scenarios" in general, to what we might call "real life." (After all, without the broader community's interest in energy in "real life," we would probably not have been supported to provide these professional development courses in the first place.) Furthermore, our epistemological view of the theory of energy, namely, that a fundamentally unproved belief in the conservation, transfer, and transformation of energy is necessary for a functional understanding of energy, suggested a limited role for logic in the induction of teachers into the (logical) use of the theory.

Our own attempted release of control over topical coverage and instructional sequence, on multiple instructional time scales, called for a new framework to be introduced into instruction in order to achieve an adequate level of discipline and accountability in classroom discourse. Through the Algebra Project [2] we found an alternative instructional method that seeks less to direct the specific content of the learner's thinking and more to regiment the relationship between that thinking and its expression and communication through multiple representations. The Algebra Project method has allowed us to meet our goals of maintaining focus on a broad overview of energy and on certain species of scientific reasoning, while capitalizing on existing community interest in energy by providing opportunity (and responsibility) for the teachers to have greater ownership of the ideas. Specifics of our adaptation of the Algebra Project instructional framework to the teaching of energy are described elsewhere [3]. 


\section{B. Learning goals}

Our goals for energy learning are specific to our population of learners and include conceptual understanding, sociopolitical relevance, creative flexibility, and representational competence.

Among the primary needs of elementary and secondary teachers is foundational conceptual understanding of physics content [4-6]. Our conceptual learning goal is for learners to conserve energy as they track the transfers and transformations of energy within, into, or out of the system of interest in complex physical processes. National standards for energy learning identify conservation and tracking as high priorities, e.g., "Students should trace where energy comes from and goes next in examples that involve several different forms of energy along the way" [7]. Similar recommendations appear in Ref. [8]. Current research in the teaching and learning of energy in biology posits tracking as the marker of high achievement in energy learning progressions [9]. These learning goals are distinct from approaches that posit energy as primarily a numerical quantity and conceptualize energy conservation in terms of mathematical balancing. In a companion paper [1], we selected a substance metaphor for energy as a primary focus of our instruction because of its advantages for teaching conservation, transfer, and flow [10-15,1].

A second need for $\mathrm{K}-12$ teachers is to make responsible connections to urgent sociopolitical issues. Teachers come to the Energy Project because they want to use science instruction to support their students in becoming good citizens, and they judge energy instruction to be particularly well suited to this purpose. Energy, as a crosscutting concept that bridges multiple sciences, contributes to explanations of phenomena in all disciplines [8]. In our courses, we strive to serve the goal of connecting energy learning to sociopolitical concerns by using complex real-world processes as contexts for analyzing energy dynamics.

Thirdly, K-12 teachers teach in a wide range of situations, with an enormous variety of populations, material resources, institutional constraints, and expectations for science learning. In order to leverage sound conceptual understanding into changes in their classrooms, teachers have to be prepared to be creative. For this reason, we work to encourage and support creative application of sound conceptual understanding in Energy Project courses. One means by which this creativity comes into play is in the invention of novel, yet potentially rigorous, representations of energy, as described in Sec. IV.

A final goal is to promote participants' competence with representations and thereby support their engagement with central practices of science. Our approach, as an adaptation of the Algebra Project, promotes and supports a rigorous correspondence between the representation of energy and observable reality. For instance, participants might agree that the box in a given scenario is moving at constant speed and therefore should be represented as having constant kinetic energy; their challenge is then to find a consistent way to show that the box has constant kinetic energy. These skills can be placed in contrast to what is probably more familiar to reform science instructors: teaching for understanding the basis for and status of scientific knowledge. These values are often exemplified by Arons' famous questions, "How do we know?" and "Why do we believe?" [16]. To highlight the contrast with our approach, we pose the different questions, "How do we show?" and "What do we believe is important?" We hope that this emphasis provides learners with access to the decisionmaking process about what basic material will constitute scientific knowledge and how scientific discourse will stand in relation to ordinary discourse.

\section{DESIGNED REPRESENTATIONS OF ENERGY: ENERGY THEATER AND ENERGY CUBES}

In designing Energy Project representations of energy, we seek to specifically harness the affordances of the energy-as-a-substance metaphor by developing representations that embody that metaphor. Since one of the most basic experiences of substances is that of object permanence, we develop representations in which energy is explicitly shown as being an object or objects. Theories of embodied cognition and cognitive linguistics suggest to us that among all possible objects, a particularly cognitively compelling sense of permanence might be attached to the self, and that use of the human body might have special significance for learning. (We summarize these theoretical contributions in Sec. III C.) Based on this synthesis of insights, we developed a representation we call "Energy Theater," in which each participant identifies as a unit of energy. Energy Theater is thus embodied in two separate senses: it uses the human body to symbolize physical entities and it makes explicit use of a particular experientially grounded metaphor (energy as a quasimaterial substance) [17].

Energy Theater is both a representation (a visual rendering of a scientific phenomenon) and a learning activity (in which participants act jointly to construct scientific ideas). In this paper, our focus is on Energy Theater as a representation. A paper in preparation focuses on Energy Theater as a learning activity [18]. Our analysis for that paper will focus on learners' interactions with one another in the material setting that Energy Theater creates.

We identify Energy Theater as a member of a class of representations that we call embodied representations, in which instructors deliberately arrange for human bodies, or parts of the body, to stand in for physics entities in the description or explanation of a phenomenon. Instructional materials that use embodied representations include Kinesthetic Astronomy [19] and sections of Physics by Inquiry [20], in which learners' bodies represent celestial bodies, certain activities in Paradigms in Physics [21] in which learners act out effective potential plots, curvilinear 
basis vectors, and charge and current density, and the Electron Runaround [22], in which learners' bodies stand in for charges in an electric circuit.

We distinguish embodied representations from other instructional activities in which the human body is employed as a physical system (rather than a symbolic system), e.g., for comparing the sensations associated with different forces, pressures, torques, and so on [23-25]. Embodied representations are also distinct from activities in which students are active for the sake of physiological stimulation. Though these activities may also enhance learning, we suggest that they do so through different mechanisms than an embodied representation does.

Below we describe Energy Theater in detail. Following that, we briefly share a second representation that is a derivative of Energy Theater, called Energy Cubes. Finally, we summarize the theoretical perspectives that suggest a special status for embodied representations such as Energy Theater.

\section{A. Energy Theater}

In Energy Theater, each participant identifies as a unit of energy that has one and only one form at any given time. Each Energy Theater enactment represents the energy transfers and transformations in a specific physical scenario (for example, a refrigerator cooling food or a light bulb burning steadily). Objects in the scenario correspond to regions on the floor. As energy moves and changes form in the scenario, participants move to different locations on the floor.

Figure 1 shows a group of participants engaged in creating an Energy Theater representation of a person pushing a box across a floor at constant speed. In this scenario, chemical energy in the hand transforms into thermal and kinetic energy as the hand moves and warms. Some kinetic energy transfers from the hand to the box, which moves. That kinetic energy is then transformed into thermal energy in the box and transferred to thermal energy in the floor as the box warms from rubbing the floor. Since the speed of the box is constant, kinetic energy transfers into the box from the hand at the same rate that it is reduced in the box via transformation to thermal energy and transfer to the environment. Each of the rules of Energy Theater is discussed below with reference to this particular group of participants.

\section{Each participant is a unit of energy}

The participants in Fig. 1 have each assumed the identity of a chunk of energy (with the exception of the seated participant in the center, who is overseeing the action). The rule that each participant is a chunk of energy (of equal amount) is vital to Energy Theater. To have each participant identify as a chunk of energy is to commit to a model of energy as a substancelike quantity. The use of one's own body promotes not only a strong commitment to conservation (people do not appear or disappear), but also transformation (in the sense that we generally retain our existence and identity while changing our age, style, etc.). Identifying as energy may also have special benefits due to learners taking the perspective of the physical system and placing participants into the sensory-motor feedback loop that the orists have argued is fundamental to human processes for meaning making (see Sec. C 3).

\section{Each unit of energy has one and only one form}

The limitation that each unit of energy has one and only one form helps participants not to "split" energy units into subunits midscenario, which can complicate issues of energy conservation. Participants decide for themselves how they will indicate the form of each unit of energy, making this step an opportunity for public construction of symbols. The participants in Fig. 1 elected to indicate form with hand signs held overhead for maximum visibility. " $\mathrm{C}$ " (chemical energy) and " $\mathrm{K}$ " (kinetic energy) were signed with hand shapes inspired by the American Manual Alphabet and " $T$ " (thermal energy) was signed with an emblem commonly indicating "time out." Advantages of this particular system include flexibility, visibility, and convenience. Disadvantages include the difficulty of

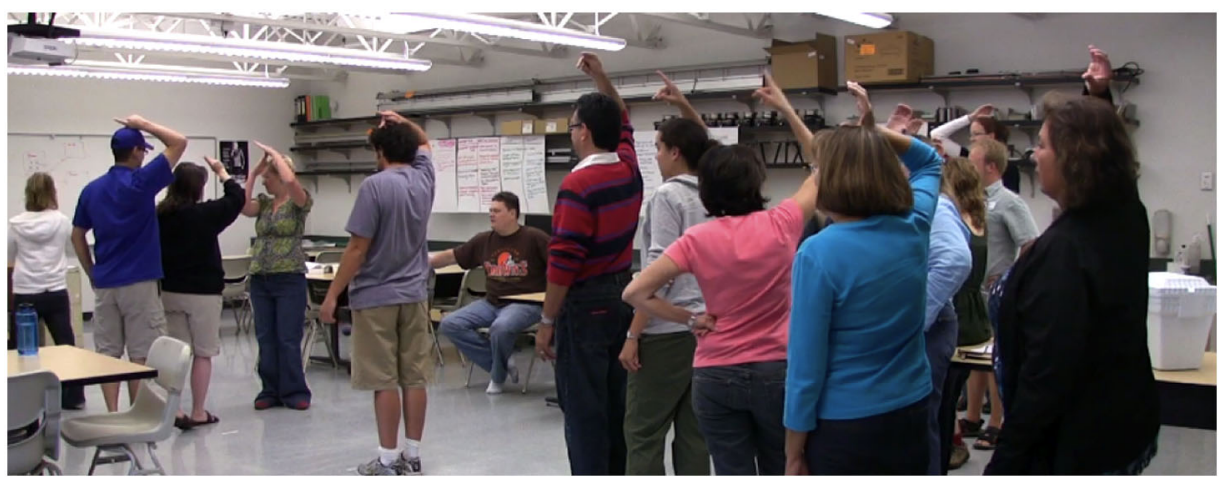

FIG. 1 (color online). Energy Theater representation of a hand pushing a box across a floor at constant speed. 
telling the signs apart at a glance, especially when many are viewed together. Other participants have used other sign systems with different advantages and disadvantages, e.g., paper signs with large letters, colored cards hung from string as a necklace, or pantomime.

\section{Objects in the scenario correspond to regions on the floor}

In Fig. 1, participants have designated areas on the floor as corresponding to the hand (where the group of people on the right is standing), the box (on the left), and the air and floor (to the far left, out of the camera frame). One participant, a male wearing shorts in Fig. 1, is on his way from the hand to the box. Participants in our courses are encouraged to designate areas on the floor with large loops of brightly colored rope.

\section{As energy moves and changes form, participants move to different regions on the floor and change their form}

This feature of Energy Theater is what makes it a dynamic representation, capable of representing energy dynamics as they unfold. It also allows comparisons of energy dynamics for different processes (e.g., how the Energy Theater representation changes if the hand pushes harder on the box).

\section{B. Energy Cubes}

The Energy Cubes representation is similar to the Energy Theater representation except that instead of chunks of energy being represented by people who move among object areas marked out on the floor, chunks of energy are represented by small cubes that move among object areas marked on a horizontal whiteboard or sheet of paper (Fig. 2). Different sides of the cubes are marked to signify different forms of energy. As

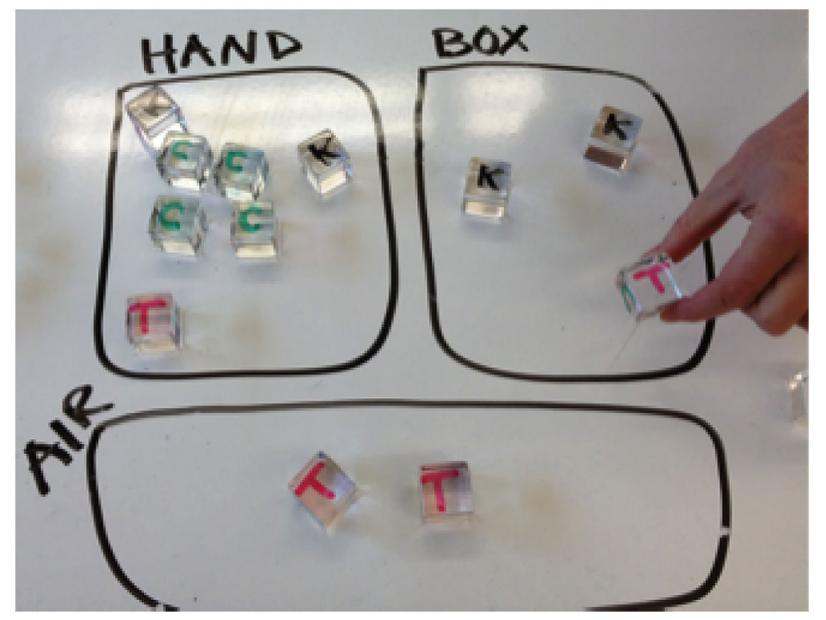

FIG. 2 (color online). Energy Cubes representation of a hand lifting a box vertically. energy transfers among and transforms within objects, users move and flip the cubes around the whiteboard. The Energy Cubes representation is similar to Feynman's description of energy as a child's blocks [26] but with added features: the location of the block shows the location of the energy and each side of the block shows a different form of the energy.

The Energy Cubes representation is also similar in some ways to a money representation, which is often suggested as a tool for teaching about energy. Like other material substances, coins and bills are localized and transferable. Money is usually thought of as being conserved in personal finance, even though in fact coins and bills are manufactured, and money is subject to inflationary processes. Money may "change forms" by changing the coins or bills that make up a given amount or by changing currency (e.g., from dollars to yen). In at least these senses, analogies between energy and money can be helpful for conveying the nature of energy. Our sense is that instructors who use this analogy do not use it to conduct detailed analysis of energy transfers and transformations or expect it to be rigorous in the sense of yielding detailed predictions. We do expect Energy Theater and Energy Cubes to bear the weight of detailed analysis, and suggest that human bodies and cubes provide more flexibility and less distraction than cash. For example, cubes have six sides that can be independently marked to signify different forms of energy. Thus, unlike money, they can change form while maintaining their identity as a specific material object.

We offer Energy Cubes as a second representational option to learners who have become comfortable with Energy Theater. Though Energy Cubes is smaller and requires fewer participants than Energy Theater, it lacks many of the cognitive affordances of an embodied representation (described in Sec. III C) and has played a more minor role in our thinking.

\section{Cognitive and interactional affordances}

Energy Theater and its derivative, Energy Cubes, strongly support a substance metaphor for energy. They represent energy as being conserved, emplaced, and changing form; they explicitly show energy as being located in objects, flowing among objects, and accumulating in objects; and they are dynamic representations, able to show processes as they unfold as well as "snapshots" of energy at specific instants. These are the features of an energy model that promote detailed tracking of energy transfers and transformations in complex real-world processes.

In addition to its advantages as a physics representation, Energy Theater has unique affordances due partly to its dependence on the human body as a representational medium. (We also understand Energy Theater to be a unique learning activity, with special affordances for 
coconstruction of scientific knowledge; we conduct that analysis in a different paper [18].) In what follows, we discuss some of the theoretical perspectives that suggest Energy Theater (and other embodied representations) are likely to be powerful for learning. In general, embodied representations support the coordination of multiple sign systems for displaying meaning, the scaling of imagined events to human size, and the symbolic identification of the self with a physics entity.

\section{Coordinating multiple sign systems for displaying meaning}

As a free multimedia technology, the human body is unsurpassed. It is not only representationally flexible, naturally dynamic, and conveniently available, but also comes with an extensive suite of tools, including gestures, vocalizations, orientations, grips, and so on. Energy Theater, like other embodied representations, promotes displays that make use of many of these tools at once. Participants in Energy Theater frequently convey meaning with words, with bodily formations (where they stand relative to other people), with motion of bodies, with hand signs for the form of energy, and with body orientation (which way they face) [27,28]. These multiple layers of representational display are not merely background features; they provide multiple ways for participants to understand one another, and are thus critical to the representation. The multiple layers of display are also relevant for assessing the learning taking place and are therefore of interest to instructors and researchers as well as fellow learners $[27,28]$. Theories of situated cognition, in which knowledge is understood to be situated in activity that is bound to social and physical contexts, can contribute to understanding such functions of Energy Theater [29-31].

\section{Scaling imagined events to human size}

The human size of the Energy Theater representation space may have implications for cognition. The theory of embodied cognition holds that because cognition takes place in terms of sensory-motor experience, we are cognitively comfortable with spatial extents and time scales that are perceptually accessible to us. Phenomena that are tiny, invisible, span millennia, etc. are understood in terms of events that are human-sized, visible, and a few seconds or minutes long. Such understandings are frequently displayed with manual gestures [32-35], consistent with the theory that cognition is not only informed by but is manifest in actions of the body [36]. Another, compatible cognitive theory, cognitive blending, argues that all learning and thinking consist of blends of metaphors based on bodily experiences [37]. In this theory, the governing principles of cognition drive toward a single overarching goal, which is to mentally convert complex and subtle experiences to human-scaled events and processes. As Lakoff and Johnson state, "human beings are evolved and culturally supported to deal with reality at human scale - that is, through direct action and perception inside familiar frames, typically involving few participants and direct intentionality" [37]. In both of these theories, part of the cognitive labor of understanding abstractions, such as energy transfers and transformations, lies in translating invisible events (such as energy dynamics) into a visible, human-sized representation. Energy Theater may be cognitively powerful to the extent that it specifically facilitates such translation. In our own research, we hope to explore the ramifications of the scaling of physical systems to the size of social systems, such that the representation space is layered onto the social-interaction space.

\section{Symbolic identification of the self with a physics entity}

The theory of embodied cognition attests that abstractions are understood in terms of basic sensory-motor experiences [38,39]. Other research in cognitive science supports a related but distinct claim: that we may leverage the embodied basis of cognition by arranging sensorymotor experiences to facilitate the learning of abstractions. Mentally placing oneself in the (perhaps symbolic) position of an entity in a physics phenomenon and symbolically reenacting the (perhaps metaphorical) movement of that entity is a key feature of expert scientific problem solving [40]. Such perspective taking is especially promoted by stable material structure in the world that stands in for the physical system under study [41], such as a graph on a blackboard [40], a shared gestural representation [32], or, we suggest, a layout of people and ropes in Energy Theater.

Experts engage in this perspective taking naturally and unconsciously [40]. With novice learners who do not engage in perspective taking automatically, we suggest that explicitly directing them to take on the role of an entity they are studying may help them to engage with the entity in a more expertlike manner and thus to understand it better. Energy Theater puts learners in the symbolic position of a unit of energy by placing them in a particular physical position in the Energy Theater arena. The arrangement prompts learners to ask themselves questions about their own location and movement, in which the learner is in the grammatical position of the unit of energy as well: "Where should I start? Should I go somewhere, or stay where I am? If I go, where should I go, and when? If I change, when and how should I change?" In such constructions, the "I" in question is neither the learner nor the energy separately, but instead blends the two [40]. Research has documented the effectiveness of a similar strategy in teaching the geometric interpretation of the addition and multiplication of complex numbers by mapping the complex plane onto the tiled classroom floor [36]. In that case, the scaling of the representation to human size 
was understood as facilitating learners' "inhabiting" the complex plane.

\section{LEARNER-INVENTED REPRESENTATIONS: TRACKING ENERGY TRANSFERS AND TRANSFORMATIONS}

Energy Theater promotes and supports a strong conceptual understanding of energy by explicitly representing energy as being conserved, being emplaced, being located in objects, flowing among objects, accumulating in objects, and changing form. Our learning goals for energy include not only a conceptual model for energy but also an analysis approach for energy dynamics: the detailed tracking of energy transfers and transformations in real-world processes. These learning goals are comparable to learning goals for Newtonian dynamics, which often include both a conceptual model for force and an analysis approach based on disciplined application of free-body diagrams.

We seek to assess whether learners have attained these conceptual and representational goals by examining the extent to which they transfer their conservation and tracking of energy to new contexts, particularly, new representational contexts. Since classic representations of energy do not strongly support both conservation and tracking, we invite learners to invent new representations of energy. We find that in response to this invitation, learners invent representations that use the substance metaphor to conserve and track energy in complex real-world scenarios. We understand this as evidence that Energy Theater is effective in promoting our learning goals.

\section{A. From embodied action to writing}

Partly because Energy Theater is a dynamic representation, it is ephemeral. An abstract symbolic system for energy can and should include a static component as well, one that can be easily recorded and shared. In courses featuring Energy Theater, participants are invited to record in writing what they did in Energy Theater. Such invitations are issued in general terms, such as, "After acting out the Energy Theater, draw and label one or more diagrams that show what happens with the energy in your scenario." Participants invent representational forms that highlight the features that are important to them and refine them in collaboration with their peers to develop diagrams that serve their needs. Figure 3 shows a sampling of diagrams for various scenarios. We will examine three of these diagrams in more detail below.

Our first observation regarding the diagrams that learners produce is their diversity. The variety in the diagrams' surface features is a testament to the learners' creativity and originality in producing the diagrams. The diagrams are entirely original in the sense that, to our knowledge, no similar diagrams appear in textbooks or in prior energy instruction that the learners may have had. We consider these diagrams to be evidence that our participants have, and are making good use of, the creativity that will be required of them to translate their energy learning into activities for their own classrooms. Research suggests that activities in which learners invent representations may have hidden efficiencies, leading to strong gains in procedural skills, insight into formulas, abilities to evaluate data from an argument, and transfer of learning to other contexts [42-44].

\section{B. Features of learner-invented representations}

Underlying the apparent variety of the diagrams, however, are deeper regularities. These regularities show that the features that learners consider important to represent are the very features that indicate a substance model for energy and enable tracking of energy transfers and transformations: i.e., energy is conserved, emplaced, located in objects, transfers among objects, accumulates in objects, and changes form. Learners who encode the deep structure of problems in selfgenerated representations are more likely to transfer their understanding of those problems to new contexts [45]. Some of the commonalities in the diagrams are

- Units of energy are shown as letters or dots. In other words, each letter or dot stands in for a person that would have taken part in Energy Theater.

- Forms of energy are indicated with letters, colors, or both. The presence of energy is indicated by the presence of letters; the form of energy is indicated by the choice of letter, e.g., $\mathrm{K}$ for kinetic or $\mathrm{T}$ for thermal.

- Objects are schematic areas (boxes or circles) or pictorial representations of the objects in the scenario.

- Time evolution is indicated with arrows or diagram sequences.

We examine three invented representations in some detail.

\section{Energy tracks, schematic objects}

Figure 3(a) shows the energy dynamics of a metal ring that is smacked by a bent-back meterstick so that it skids across the floor. In this diagram, objects are schematic areas (loops), and each energy unit is a letter that traces a path through the system of objects. This representational system matches closely to Energy Theater; each letter stands in for a person (unit of energy), each loop stands for a loop of rope (object), and each energy unit is tracked as it changes form and transfers among objects in the system. However, it is also significantly different from Energy Theater in that the entire sequence of energy transfers and transformations is represented on a single diagram rather than being shown in succession. Energy conservation is naturally enforced as letters are sustained along their tracks. In this particular diagram, the ring and ruler are pictured as a single object within which energy transforms from potential (P) to motion (M) energy. Some of the motion energy is transferred to the floor and becomes thermal (T) and sound 

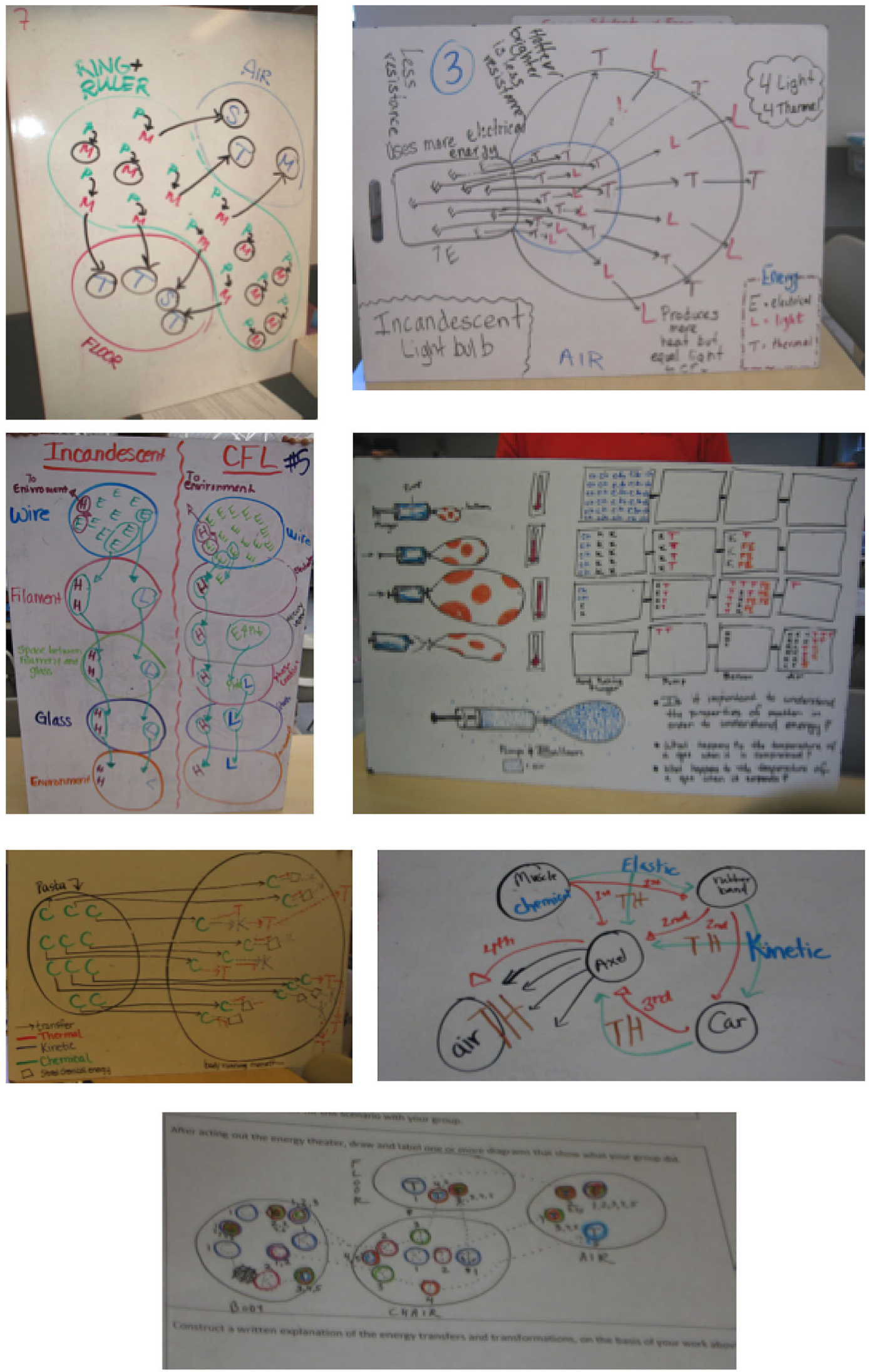

FIG. 3 (color online). Learner-invented representations that track energy transfers and transformations in (a) a ring smacked across the floor by a bent-back meterstick, (b) an incandescent light bulb burning steadily, (c) incandescent and compact fluorescent light bulbs, (d) a pumped balloon, (e) a runner eating pasta, (f) a pullback car, (g) a person pushing a chair. 
(S) energy, some motion energy goes to the air as sound, thermal, and motion energy, and some stays in the ring and ruler.

\section{Energy tracks, pictorial objects}

Figure 3(b) illustrates the energy dynamics of an incandescent light bulb burning steadily. In this diagram, as in Fig. 3(a), each energy unit is a letter that traces a path through the system. Each unit of energy is traced along a specific path among the objects in the scenario (light bulb base, filament, glass bulb, and surrounding air). At the approximate location along its path where the form of energy changes, the letter changes (e.g., from an E to an $\mathrm{L}$ ). This diagram models the energy dynamics approximately as follows: Electrical energy in the base of the bulb travels to the filament, where it is transformed into thermal and light energy; these forms of energy travel out through the bulb to the surrounding air. This model, though incomplete, has the important properties of the substance model: Energy is shown as located in objects, transferring among objects, transforming among forms, and being conserved. This diagram is a faithful reproduction of the Energy Theater performed by the group that created it, including the layout of ropes in pictorial rather than schematic form. This representational choice, though unanticipated by the instructors, does not appear to hinder, and in fact may assist, the learners in relating energy dynamics to real physical phenomena.

\section{Energy snapshots, schematic objects}

Figure 3(d) shows the energy dynamics of a pumped balloon. In this case, energy units are colored letters and objects are schematic areas (from left to right: "hand pushing plunger," "pump," "balloon," and "air"). Successive rows of the diagram are successive moments in the process of filling the balloon; in other words, the diagram represents "snapshots" of the energy distribution at particular instants, rather than tracing the path of specific energy units through a system. In this representation, energy conservation has to be intentionally enforced by counting the total number of energy units (letters) at each time (in each row). An additional feature of this diagram is that the distribution of energy at given moments in the process is coordinated with the observable state of the system at those moments (plunger position, balloon volume, and temperature). The process represented is approximately as follows: Chemical energy in the hand is transformed into kinetic energy in the hand and transferred to (1) kinetic and thermal energy in the pump and (2) kinetic, thermal, and (presumably elastic) potential energy in the balloon. As the balloon is pumped to its maximum, the hand's chemical and kinetic energy decrease and the balloon gains more thermal and potential energy. When the balloon detaches from the pump, most of the energy winds up as kinetic and thermal energy in the air. This representation also has the important properties of the substance model: Energy is represented as being located in objects, transferring among objects, transforming among forms, and being conserved.

\section{EXPERT-REFINED REPRESENTATIONS: TOOLS FOR CONTENT UNDERSTANDING, TEACHING, AND RESEARCH}

The diagrams above are powerful representations of energy. They are not merely a creative outlet or recordkeeping chore for the learners that use them, but are rather a serious contribution to the representational options for energy in physical systems, promoting specific, complex analysis of energy transfers and transformations in a way that pie charts and bar graphs do not. The questions such diagrams ask are the questions we want learners to ask, and indeed are the questions we ask ourselves when analyzing energy scenarios: "Where is the energy when the physical process in question begins? Where does it go after that? What form is each unit of energy in at each moment?" We find these representations so compelling that we have refined a few of them for our own use, both in teaching and in analysis of energy dynamics for our own physics understanding. These refined diagrams are also powerful tools for researchers, who can use the representation to "code" video of teachers doing Energy Theater, highlighting the energy dynamics represented and temporarily concealing the interpersonal dynamics of the participants. We describe two different expert-refined diagrams below.

\section{A. Energy Tracking Diagram}

An Energy Tracking Diagram is similar to the diagram described above for the smacked ring. An example of an Energy Tracking Diagram for a hand compressing a spring at constant speed is shown in Fig. 4. In this scenario, chemical energy in the hand transforms into thermal and kinetic energy (the hand moves and warms). Some of the thermal energy in the hand is transferred to the
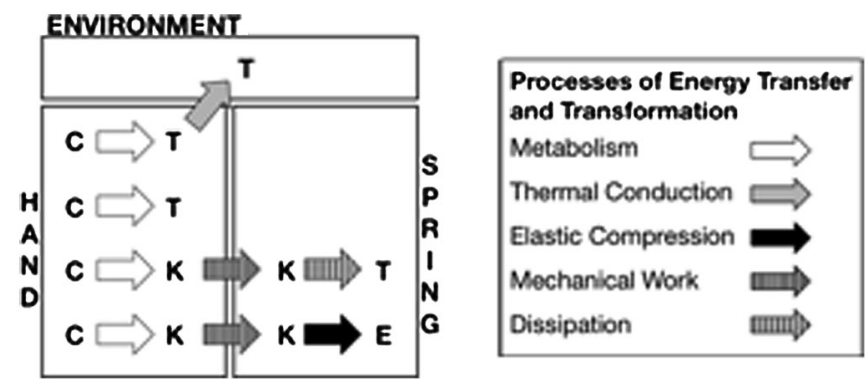

FIG. 4. Energy Tracking Diagram for a hand compressing a spring at constant speed. C, K, T, and E represent chemical, kinetic, thermal, and elastic energy, respectively. White, gray, black, striped, and hatched arrows represent metabolism, thermal conduction, elastic compression, mechanical work, and dissipation, respectively. 
environment via conduction. Some kinetic energy transfers to the spring, which moves; that kinetic energy is then transformed into elastic energy and thermal energy as the spring compresses and warms. The rules of an Energy Tracking Diagram are as follows:

- Objects are represented as schematic areas.

- Individual units of energy are represented as individual letters, with the specific letter representing the form of energy.

- Energy transfers and transformations are represented with arrows. All arrows have a letter at the head and the tail. Arrows that cross the boundaries of object areas indicate energy transfers. Arrows that have a different letter at the head than the tail indicate energy transformations.

- The process by which a transfer or transformation occurs (e.g., work, metabolism, conduction) is indicated by the color or pattern of the arrow. For example, in Fig. 4, striped arrows indicate mechanical work and gray arrows indicate thermal conduction.

- Time order of energy transfers and transformations is represented by sequences of arrows. (The time order of processes that occur along separate tracks is not represented.)

- Relative amounts of energy may be represented by adding coefficients to the letters representing units of energy. (Figure 4 does not include such coefficients.)

\section{B. Energy Animation}

The refined representation that we call an energy animation is more like Energy Theater itself in that it is a dynamic representation, but is similar in other ways to an Energy Tracking Diagram. The rules of an energy animation are as follows:

- Objects are represented as schematic areas in a medium that permits simple animation, such as commercial presentation software.

- Individual units of energy are represented as individual letters, with the specific letter indicating the form of energy.

- Energy transfer is represented by a letter traveling from one object area to another. Energy transformation is represented by a letter changing into a different letter. (The process by which a transfer or transformation occurs is not represented.)

- Time order of energy transfers and transformations is represented faithfully in the animation.

Energy animations have the important advantage of being dynamic. However, because significant time is required to prepare an energy animation (especially for users without specialized skills or programming expertise), this representation is best suited to instructional demonstrations prepared in advance.

\section{SUMMARY}

The invention and refinement of representations is a central activity in the construction of scientific knowledge. We use a novel representation that embodies the substance metaphor, in which each participant identifies as a unit of energy that has one and only one form, objects in a physical scenario correspond to regions on the floor, and participants move around on the floor to represent transfers of energy among objects, changing their hand sign or other marker to indicate changes of form. This embodied representation, Energy Theater, supports the coordination of multiple sign systems for displaying meaning, the scaling of physical systems to human size, and the symbolic identification of the self with a physics entity.

Learners who are taught Energy Theater subsequently invent graphical representations that articulate a conceptualization in which energy is conserved, localized, and located in objects, flows among objects, accumulates in objects, and changes form. These representations constitute a significant contribution to the representational repertoire for energy in physical systems, with specific advantages over other representations for tracking energy transfers and transformations. Learner-invented derivatives of Energy Theater are powerful enough that we have adapted some of them for our own use, both in teaching about energy and in analyzing the energy dynamics of physical scenarios for our own understanding.

\section{ACKNOWLEDGMENTS}

We gratefully acknowledge all the elementary and secondary teachers who have participated in Energy Project courses for their generosity in making their own and their students' reasoning accessible to the Energy Project team. We are indebted to all the members of the Physics Education Research Group at Seattle Pacific University, especially Lezlie S. DeWater, Sarah B. McKagan, and Lane Seeley for their substantial contributions to the development of Energy Theater and the course design that supported this research, and Abigail R. Daane for contributions to the development of Energy Tracking Diagrams. We also thank the national community of Energy Project collaborators for their engagement with our work, particularly Leslie J. Atkins, Benedikt Harrer, and Jim Minstrell. This material is based upon work supported by the National Science Foundation under Grant No. 0822342. 
[1] R. E. Scherr, H. G. Close, S. B. McKagan, and S. Vokos, Representing energy. I. Representing a substance ontology for energy, preceding paper, Phys. Rev. ST Phys. Educ. Res. 8, 020114 (2012).

[2] R.P. Moses and C.E. Cobb, Radical Equations: Civil Rights from Mississippi to the Algebra Project (Beacon, Boston, MA, 2001).

[3] H. G. Close, L. S. DeWater, E. W. Close, R. E. Scherr, and S. B. McKagan, Using the Algebra Project method to regiment discourse in an energy course for teachers, AIP Conf. Proc. 1289, 9 (2010).

[4] L.C. McDermott and L.S. DeWater, in The need for special science courses for teachers: Two perspectives, in Inquiring into Inquiry in Science Learning and Teaching, edited by J. Minstrell and E. van Zee (AAAS, Washington, DC, 2000).

[5] P. S. Shaffer and L. C. McDermott, A research-based approach to improving student understanding of kinematical concepts, Am. J. Phys. 73, 921 (2005).

[6] L. C. McDermott, P.S. Shaffer, and C.P. Constantinou, Preparing teachers to teach physics and physical science by inquiry, Phys. Educ. 35, 411 (2000).

[7] American Academy for the Advancement of Science, Benchmarks for Science Literacy (Oxford University Press, New York, 1993).

[8] National Research Council, A Framework for $\mathrm{K}-12$ Science Education: Practices, Crosscutting Concepts, and Core Ideas (National Academies Press, Washington, DC, 2012).

[9] H. Jin and C.W. Anderson, Learning progression for energy in carbon-transforming processes (unpublished).

[10] A. diSessa, Ontologies in pieces: Response to Chi and Slotta, Cogn. Instr. 10, 272 (1993).

[11] R. Duit, Should energy be illustrated as something quasimaterial?, Int. J. Sci. Educ. 9, 139 (1987).

[12] G. Swackhamer, Cognitive resources for understanding energy, http://modeling.asu.edu/modeling/CognitiveResourcesEnergy.pdf.

[13] E. Brewe, Energy as a substancelike quantity that flows: Theoretical considerations and pedagogical consequences, Phys. Rev. ST Phys. Educ. Res. 7, 020106 (2011).

[14] R. Millar, "Teaching about energy," Department of Educational Studies Research Paper, York University, 2005).

[15] G. Falk, F. Herrmann, and G. Bruno Schmid, Energy forms or energy carriers?, Am. J. Phys. 51, 1074 (1983).

[16] A. B. Arons, Achieving wider scientific literacy, Daedalus (Boston) 112, 91 (1983).

[17] R. Stevens, The missing bodies of mathematical thinking and learning have been found, J. Learn. Sci. 21, 337 (2012).

[18] R. E. Scherr et al., "Understanding energy with a social embodied learning activity" (to be published).

[19] C. A. Morrow, Kinesthetic Astronomy: The sky time lesson, Phys. Teach. 38, 252 (2000).

[20] L.C. McDermott, P.S. Shaffer, and M. L. Rosenquist, Physics by Inquiry: An introduction to Physics and the Physical Sciences (John Wiley \& Sons, New York, 1996).
[21] C. A. Manogue, P. J. Siemens, J. Tate, K. Browne, M.L. Niess, and A. J. Wolfer, Paradigms in Physics: A new upper-division curriculum, Am. J. Phys. 69, 978 (2001).

[22] V. Singh, The Electron Runaround: Understanding electric circuit basics through a classroom activity, Phys. Teach. 48, 309 (2010).

[23] H. Pfister and P. Laws, Kinesthesia-1: Apparatus to experience 1-D motion, Phys. Teach. 33, 214 (1995).

[24] P. Pantidos and S. Patapis, Kinesthetic transverse wave demonstration, Phys. Teach. 43, 344 (2005).

[25] H. G. Close and P. R. L. Heron, Student understanding of the angular momentum of classical particles, Am. J. Phys. 79, 1068 (2011).

[26] R. P. Feynman, R. B. Leighton, and M. Sands, The Feynman Lectures on Physics (Addison-Wesley, Reading, MA, 1969).

[27] C. Goodwin, Action and embodiment within situated human interaction, J. Pragmatics 32, 1489 (2000).

[28] E. W. Close, H. G. Close, S. B. McKagan, and R. E. Scherr, Energy in action: The construction of physics ideas in multiple modes, AIP Conf. Proc. 1289, 105 (2010).

[29] E. Hutchins, Cognition in the Wild (MIT Press, Cambridge, MA, 1995).

[30] J.G. Greeno, The situativity of knowing, learning, and research, Am. Psychol. 53, 5 (1998).

[31] J. Lave, Situating learning in communities of practice, in Perspectives on Socially Shared Cognition, edited by L. B. Resnick, J. M. Levine, and S. D. Teasley (American Psychological Association, Washington, DC, 1991), pp. 63-82.

[32] L. A. Becvar, J. Hollan, and E. Hutchins, Hands as molecules: Representational gestures used for developing theory in a scientific laboratory, Semiotica 156, 89 (2005).

[33] M. W. Alibali, M. Bassok, K. O. Solomon, S. E. Syc, and S. Goldin-Meadow, Illuminating mental representations through speech and gesture, Psychol. Sci. 10, 327 (1999).

[34] W.-M. Roth and D. Lawless, Scientific investigations, metaphorical gestures, and the emergence of abstract scientific concepts, Learn. Instr. 12, 285 (2002).

[35] R.E. Scherr, Gesture analysis for physics education researchers, Phys. Rev. ST Phys. Educ. Res. 4, 010101 (2008).

[36] R. Nemirovsky, C. Rasmussen, G. Sweeney, and M. Wawro, When the classroom floor becomes the complex plane: Addition and multiplication as ways of bodily navigation, J. Learn. Sci. 21, 287 (2012).

[37] G. Fauconnier and M. Turner, The Way We Think: Conceptual Blending and the Mind's Hidden Complexities (Basic Books, New York, 2002).

[38] G. Lakoff and M. Johnson, Philosophy in the Flesh: The Embodied Mind and its Challenge to Western Thought (Basic Books, New York, 1999).

[39] G. Lakoff and R. E. Nuñez, Where Mathematics Comes From: How the Embodied Mind Brings Mathematics into Being (Basic Books, New York, 2000). 
[40] E. Ochs, P. Gonzales, and S. Jacoby, "When I come down I'm in the domain state": Grammar and graphic representation in the interpretive activity of physicists, in Interaction and Grammar, edited by E. Ochs, E. Schegloff, and S. Thompson (Cambridge University Press, Cambridge, England, 1996), pp. 328-369.

[41] E. Hutchins, Material anchors for conceptual blends, J. Pragmatics 37, 1555 (2005).

[42] N.S. Podolefsky and N.D. Finkelstein, Analogical scaffolding and the learning of abstract ideas in physics: An example from electromagnetic waves, Phys. Rev. ST Phys. Educ. Res. 3, 010109 (2007).
[43] N. S. Podolefsky and N. D. Finkelstein, Analogical scaffolding and the learning of abstract ideas in physics: Empirical studies, Phys. Rev. ST Phys. Educ. Res. 3, 020104 (2007).

[44] D. L. Schwartz and T. Martin, Inventing to prepare for future learning: The hidden efficiency of encouraging original student production in statistics instruction, Cogn. Instr. 22, 129 (2004).

[45] D. L. Schwartz, "Why direct instruction earns a C- in transfer," Northwestern Center for Engineering Education Research (unpublished). 